\title{
Quark mass dependence of the pion form factor
}

\author{
Felipe J. Llanes-Estrada* fllanes@fis.ucm.es \\ Feng-Kun Guo ${ }^{\dagger}$, Christoph Hanhart ${ }^{\dagger, \%}$ and Ulf-G. Meißner ${ }^{\dagger}, \%, \&$ \\ * Universidad Complutense de Madrid, Fisica Teorica I, 28040 Madrid \\ ${ }^{\dagger}$ Institut für Kernphysik and Jülich Center for Hadron Physics, Forschungszentrum Jülich, \\ D-52425 Jülich, Germany \\ \% Institute for Advanced Simulations, Forschungszentrum Jülich, D-52425 Jülich, Germany \\ ${ }^{\&}$ Helmholtz-Institut für Strahlen- und Kernphysik (Theorie) and Bethe Center for Theoretical \\ Physics, Universität Bonn, D-53115 Bonn, Germany
}

\begin{abstract}
We present a first study of the quark mass dependence of the pion vector form factor, particularly its curvature (mean quartic radius). By employing the Omnès representation we can provide a very clean estimate for a certain combination of the curvature and the square radius, whose quark mass dependence is being determined from lattice computations. This currently requires an extrapolation to the physical point. The reach of validity of this extrapolation is determined by the appearance of the first non-analyticity in the form factor as function of the quark mass. We also provide an improved value for the curvature at physical values of the quark masses, namely $\left\langle r^{4}\right\rangle=0.73 \pm 0.09 \mathrm{fm}^{4}$ or equivalently $c_{V}=4.00 \pm 0.50 \mathrm{GeV}^{-4}$, for Unitarized Chiral Perturbation Theory, and $\left\langle r^{4}\right\rangle=0.68 \pm 0.06 \mathrm{fm}^{4}, c_{V}=3.75 \pm 0.33 \mathrm{GeV}^{-4}$, for a Breit-Wigner parametrization of the pion scattering phase shifts used in the Omnès representation.
\end{abstract}

International Workshop on Effective Field Theories: from the pion to the upsilon 2-6 February 2009

Valencia, Spain

* Speaker. 


\section{Introduction}

A low-momentum expansion of the pion form factor defines its square and quartic radii,

$$
F(t)=1+\frac{1}{3 !}\left\langle r^{2}\right\rangle t+\frac{1}{5 !}\left\langle r^{4}\right\rangle t^{2}+\mathscr{O}\left(t^{3}\right)
$$

the latter also called curvature [1] with the redefinition $c_{V}^{\pi}=\frac{1}{5 !}\left\langle r^{4}\right\rangle$. These quantities are now being calculated in Lattice Gauge Theory for large values of the light quark masses (equivalently, pion mass) that allow extrapolation to the physical point aided by Chiral Perturbation Theory $(\chi P T)$. While the square radius is NLO in $\chi P T$, the curvature is itself NNLO in the expansion, and a study of this with controlled uncertainty would require knowledge at NNNLO, which seems out of today's reach. We therefore adopt the Omnès representation of the form factor, that, for physical pion mass, requires only a parametrization of the scattering $\delta_{11}$ phase shift. Employing Unitarized Chiral Perturbation Theory, also called inverse amplitude method (IAM), for this phase shift, we can control the mass-dependence of the resulting form factor, the result being rigorous to first order in the mass expansion. Alternatively we calculate the same quantity using a Breit-Wigner parametrization for the phase shifts accompanied by the quark mass dependence of the $\rho$ mass as deduced from different sources [2] and the additional assumption that the $\rho \pi \pi$ coupling is independent of the quark mass. Our study has recently been presented in [3], to which we refer for further detail and bibliography.

\section{The Omnès representation}

The Omnès equation encodes the analyticity properties of the pion form factor $F(s)$, that has an elastic unitarity cut on the positive $s$-axis for $s \in\left(4 m_{\pi}^{2}, \infty\right)$, and is otherwise analytic. Further superimposed cuts due to inelastic channels are neglected in its derivation, and the form factor is assumed to have no zeroes (which, as we know today, is phenomenologically correct). The starting point is the well known relation $\operatorname{Im}(F)=\tan \delta_{11} \operatorname{Re}(F)$, which relates the discontinuity in the vector form factor to the elastic scattering phase shift in the vector-isovector channel. From this relation the Watson theorem follows straightforwardly. Since the large- $q^{2}$ asymptotic behavior of the form factor is known from QCD counting rules, $F\left(q^{2}\right) \rightarrow c / q^{2}$, as a matter of principle one may write an unsubtracted dispersion relation, which reads for arbitrary $t$

$$
F(t)=\frac{1}{\pi} \int_{4 m_{\pi}^{2}}^{\infty} d s \tan \delta_{11}(s) \frac{\operatorname{Re}(F(s))}{s-t-i \varepsilon} .
$$

We specified "as a matter of principle" since the QCD counting rules apply only when elastic scattering is irrelevant by the numerous inelastic channels open. However, in this work we only want to use low energy input up to $1.2 \mathrm{GeV}$ and we will therefore use a subtracted dispersion relation below and cut the high energy contributions with a cut-off. The variation of the results with this cut-off provides a systematic uncertainty in our work, which, as a consequence of the subtraction, turns out to be moderate.

If there are no bound state poles, as is the case of $\pi \pi$ scattering for physical quark masses, nor the form factor vanishes anywhere in the complex plane, as we presume for $F(t)$, the celebrated 
solution family of this equation provides a representation of the form-factor in terms of the scattering phase shifts, known as the Omnès representation. The standard treatment proceeds by deriving an integral equation for $\log F(t) /(2 i)$ instead of $F(t)$ itself,

$$
\log \frac{F(t)}{2 i}=\frac{1}{2 \pi i} \int_{4 m_{\pi}^{2}}^{\infty} \frac{d s}{s-t}\left(\log \frac{F(s+i \varepsilon)}{2 i}-\log \frac{F(s-i \varepsilon)}{2 i}\right)=\frac{1}{\pi} \int_{4 m_{\pi}^{2}}^{\infty} \frac{d s}{s-t} \delta_{11}(s) .
$$

Instead of this relation we may use a subtracted version. This will allow us to effectively suppress the high energy behavior of the phase shifts. In particular we will use a twice subtracted version which reads after exponentiation

$$
F(t)=\exp \left(P_{1} t+\frac{t^{2}}{\pi} \int_{4 m_{\pi}^{2}}^{\infty} d s \frac{\delta_{11}(s)}{s^{2}(s-t-i \varepsilon)}\right) .
$$

Note, the normalization condition of the form factor prohibits a constant term in the exponent. The constant $P_{1}$ can be identified with the square radius of the pion, $P_{1}=\left\langle r^{2}\right\rangle / 6$.

We may read off an expression for $c_{V}^{\pi}$ directly from Eq. (2.3):

$$
c_{V}^{\pi}=\frac{\left\langle r^{4}\right\rangle}{120}=\frac{1}{72}\left\langle r^{2}\right\rangle^{2}+\frac{1}{\pi} \int_{4 m_{\pi}^{2}}^{\infty} d s \frac{\delta_{11}(s)}{s^{3}}
$$

which is quite a beautiful formula, since it allows a third independent extraction of the curvature $c_{V}^{\pi}$ besides NNLO $\chi \mathrm{PT}$ or a fit to spacelike data beyond the linear fall in $t$ where uncertainties get large (more sophisticated dispersive analysis [4] show the continuous interest in this curvature). Instead we employ only the elastic phase shifts. In addition, since the quantity

$$
\tilde{c}_{V}^{\pi} \equiv c_{V}^{\pi}-\frac{1}{72}\left\langle r^{2}\right\rangle^{2}
$$

is described solely in terms of the $\pi \pi p$-wave phase shifts, its quark mass dependence is linked to that of the $\rho$-meson properties.

\section{Chiral perturbation theory and curvature computation}

We start by recalling the chiral expansion of the vector form factor valid to NLO in $\chi \mathrm{PT}$,

$$
F(t)=1+\frac{1}{6 f_{\pi}^{2}}\left(t-4 m_{\pi}^{2}\right) \bar{J}(t)+\frac{t}{96 \pi^{2} f_{\pi}^{2}}\left(\bar{l}_{6}-\frac{1}{3}\right) .
$$

Here

$$
\bar{J}(t)=\frac{1}{16 \pi^{2}}\left[\sigma \log \left(\frac{\sigma-1}{\sigma+1}\right)+2\right]
$$

with $\sigma=\sqrt{1-4 m_{\pi}^{2} / t}$. A common strategy is to fix the $\bar{l}_{6}$ constant from the square charge radius

$$
\left\langle r^{2}\right\rangle=\frac{1}{16 \pi^{2} f_{\pi}^{2}}\left(\bar{l}_{6}-1\right)
$$

which is correct up to $\mathscr{O}\left(m_{\pi}^{2}\right)$ in $\chi \mathrm{PT}$. Higher orders in the chiral expansion cannot bring in powers of $t$ since, by definition, the charge squared radius is proportional to the coefficient of the term 
linear in $t$ in the form factor. However, they can bring additional constants to the right hand side (each of a natural order suppressed by additional factors of $1 /\left(4 \pi f_{\pi}\right)^{2}$ ), and, more important for our purposes, a polynomial of $m_{\pi}^{2}$. To make sure we are not eschewing a critical $m_{\pi}$ dependence, we will compare the right-hand-side of eq.(3.3) with the NNLO correction in chiral perturbation theory [6]. The NLO result eq. (3.3), that depends only logarithmically on the pion mass (see eq. (3.6) below), is then extended to

$$
\begin{array}{r}
\left\langle r^{2}\right\rangle=\frac{1}{16 \pi^{2} f_{\pi}^{2}}\left[\left(1+\frac{m_{\pi}^{2}}{8 \pi^{2} f_{\pi}^{2}} \bar{l}_{4}\right)\left(\tilde{l}_{6}-1\right)+\frac{m_{\pi}^{2}}{16 \pi^{2} f_{\pi}^{2}}\left(16 \pi^{2} \frac{13}{192}-\frac{181}{48}\right)\right] \\
\tilde{l}_{6} \equiv \bar{l}_{6}+6 \frac{m_{\pi}^{2}}{f_{\pi}^{2}}\left[16 \pi^{2} r_{V 1}^{r}\left(\mu^{2}\right)+\frac{1}{48 \pi^{2}} \log \left(\frac{m_{\pi}^{2}}{\mu^{2}}\right)\left(\frac{19}{12}-\bar{l}_{1}+\bar{l}_{2}\right)\right]
\end{array}
$$

where $r_{V 1}^{r}$ is a counterterm to be determined empirically, and we will use the simple VMD estimate from the same work, at the $\rho$ scale, $r_{V 1}^{r}\left(m_{\rho}^{2}\right) \simeq-0.25 \times 10^{-3}$. Then $\tilde{l}_{6}=\bar{l}_{6}-1.44$ (the scaledependence of this number cancels).

Next we recall the pion mass dependence of the $\bar{l}$ 's. The $l_{i}$, as coefficients of the expansion in powers of $m_{\pi}^{2}$ of the Lagrangian density, are by definition pion-mass independent, and so are their renormalized counterterms $l_{i}^{r}$. However, the barred quantities absorb a chiral logarithm

$$
l_{i}^{r}=\frac{\gamma_{i}}{32 \pi^{2}}\left[\bar{l}_{i}+\log \left(\frac{m_{\pi}^{2}}{\mu^{2}}\right)\right]
$$

that makes the $\bar{l}$ 's scale-independent, but in exchange, $m_{\pi}$-dependent. This dependence needs to be kept track of in the calculation. This becomes crucial in the chiral limit when the pion radius diverges due to the virtual pion cloud becoming long-ranged as the pion mass vanishes.

We thus denote by $l_{i}^{\text {phys }}$ the value that the low energy constants take by fitting to physicalworld data. Henceforth, when varying $m_{\pi}$, one needs to change the constant according to

$$
\bar{l}_{i}=\bar{l}_{i}^{\text {phys }}-\log \left(\frac{m_{\pi}^{2}}{\left(m_{\pi}^{\text {phys }}\right)^{2}}\right)
$$

From $\chi$ PT we also take the quark-mass dependence of $\left\langle r^{2}\right\rangle-$ c.f. Eq. (2.3). Clearly, the curvature $c_{V}^{\pi}$ could also be determined in $\chi \mathrm{PT}$ directly. Depending on the fit and systematics chosen in Ref. [6], a two-flavor $\mathscr{O}\left(p^{6}\right) \chi \mathrm{PT}$ calculation, its value could vary between $2-6 \mathrm{GeV}^{-4}$, although the authors quote a value around $2.25 \mathrm{GeV}^{-4}$, in agreement with previous estimates. By fitting to form factor data, they obtain $3.85 \mathrm{GeV}^{-4}$ ).

With these mass-dependences, one can employ a Breit-Wigner parametrization of $\delta_{11}$,

$$
\delta_{11}(s)=\arctan \frac{\operatorname{Im} a_{11}(s)}{\operatorname{Re} a_{11}(s)}=\arctan \frac{m_{\rho} \Gamma_{\text {tot }}(s)}{m_{\rho}^{2}-s}
$$

in terms of the $\rho$ mass and total width, $\Gamma_{\text {tot }}=\frac{g_{\rho \pi \pi}^{2} p^{3}}{6 \pi m_{\rho}^{2}}=\frac{g_{\rho \pi \pi}^{2}\left(\frac{s}{4}-m_{\pi}^{2}\right)^{3 / 2}}{6 \pi m_{\rho}^{2}}$, and study $F(t)$ for unphysical pion masses [3] using the input quoted above.

For the IAM one can estimate the quark mass dependence of the $\rho$ properties directly from the $\chi \mathrm{PT}$ amplitudes evaluated up to a given order. It implements NLO in $\chi \mathrm{PT}$ at low momentum, satisfies elastic unitarity exactly, and fits the pion scattering data up to $1.2 \mathrm{GeV}$ well. To derive the 

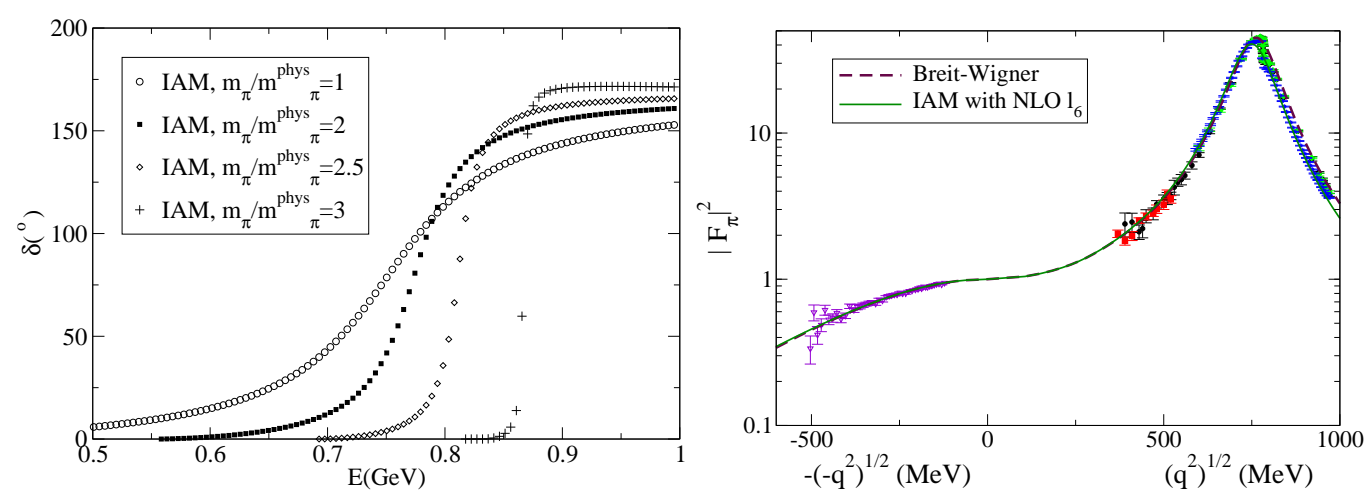

Figure 1: Left: Variation of the elastic $\pi \pi$ phase $\delta_{11}$ with $m_{\pi}$ in the IAM. The resonance stays above the $\pi \pi$ threshold, its mass having a slight dependence on $m_{\pi}$, until rather high $m_{\pi}$. Right: Square form factor modulus for both spacelike and timelike $q^{2}$, fitted with the Omnès representation.

expression for the IAM one starts with the on-shell $\pi \pi$ scattering amplitude in NLO $\chi \mathrm{PT}$ that, for $I=1$, is $A_{1}(s, t, u)=A(t, s, u)-A(u, t, s)$, with

$$
\begin{aligned}
A(s, t, u) & =\frac{s-m_{\pi}^{2}}{F^{2}}+\frac{1}{6 F^{4}}\left[3 \bar{J}(s)\left(s^{2}-m_{\pi}^{4}\right)+\bar{J}(t)\left(t(t-u)-2 m_{\pi}^{2} t+4 m_{\pi}^{2} u-2 m_{\pi}^{4}\right)\right. \\
& \left.+\bar{J}(u)\left(u(u-t)-2 m_{\pi}^{2} u+4 m_{\pi}^{2} t-2 m_{\pi}^{4}\right)\right]+\frac{1}{96 \pi^{2} f_{\pi}^{4}}\left[2\left(\bar{l}_{1}-\frac{4}{3}\right)\left(s-2 m_{\pi}^{2}\right)^{2}\right. \\
& \left.+\left(\bar{l}_{2}-\frac{5}{6}\right)\left(s^{2}+(t-u)^{2}\right)-3 m_{\pi}^{4} \bar{l}_{3}-12 m_{\pi}^{2} s+15 m_{\pi}^{4}\right] .
\end{aligned}
$$

The first term can be identified as the leading order, low-energy theorem, but we express it in terms of the physical $m_{\pi}$, instead of its leading order value $M$. Meanwhile, we keep the $m_{\pi}$ independent pion decay constant $F$. The quantities $F$ and $M$ are related to the physical ones via

$$
F=f_{\pi}\left(1-\frac{m_{\pi}^{2}}{16 \pi^{2} f_{\pi}^{2}} \bar{l}_{4}\right), \quad M^{2}=m_{\pi}^{2}\left(1+\frac{m_{\pi}^{2}}{32 \pi^{2} f_{\pi}^{2}} \bar{l}_{3}\right) .
$$

The latter expression introduces $\bar{l}_{3}$ into the last line of Eq. (3.8).

The projection to the spatial $p$-wave has the usual factor of $1 / 2$ to avoid double-counting quantum states by counting all angular configurations with exchanged identical particles

$$
a_{11}(s)=\frac{1}{32 \pi} \frac{1}{2} \int_{-1}^{1} d \cos \theta(\cos \theta) A_{1}(s, t(s, \cos \theta), u(s, \cos \theta)) .
$$

One can organize the chiral expansion as $a_{11}(s)=a_{11}^{\mathrm{LO}}(s)+a_{11}^{\mathrm{NLO}}(s)+\ldots$ but the series truncated at any order only satisfies elastic unitarity perturbatively. This is solved, with the first two expansion terms, by the IAM [7] that reads (suppressing the spin and isospin subindices)

$$
a^{\mathrm{IAM}}(s)=\frac{a_{\mathrm{LO}}^{2}(s)}{a^{\mathrm{LO}}(s)-a^{\mathrm{NLO}}(s)} .
$$

The first two terms of a Taylor expansion of this amplitude returns NLO $\chi$ PT as usual for a Padé approximant. However elastic unitarity is now exact, and the possibility of a zero of the denominator allows for resonances. 

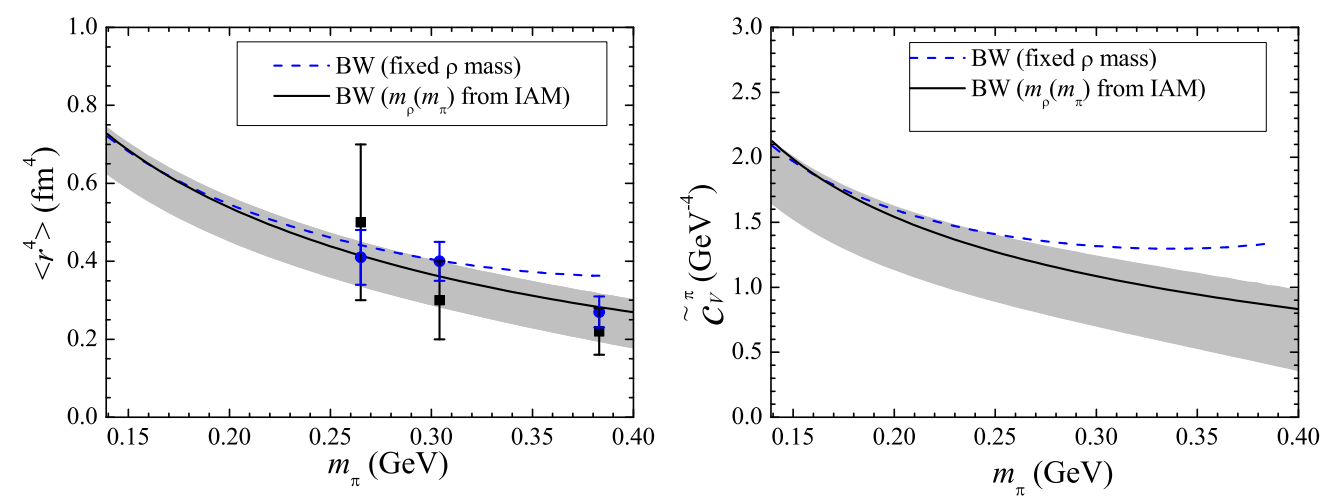

Figure 2: Left: $m_{\pi}$ dependence of the quartic radius for moderate pion masses, plotted together with a new lattice computation[5]. Right: $\tilde{c}_{V}$, the Omnès integral over the $\rho$ phase shift, directly accessible to the lattice as a combination of quadratic and quartic spacelike radii. Lines: Breit-Wigner results. Band: IAM.

The associated phase shift

$$
\delta_{11}^{\mathrm{IAM}}(s)=\arctan \left(\frac{\operatorname{Im} a_{11}^{\mathrm{IAM}}(s)}{\operatorname{Re} a_{11}^{\mathrm{IAM}}(s)}\right)
$$

may be directly employed for the time-like form factor through the Omnès representation.

The low energy constants necessary to complete the calculation are fit to the phase shift data with fixed $\bar{l}_{3}=2.9$, obtaining the values $\bar{l}_{1}=0.1 \pm 1.5, \bar{l}_{2}=6 \pm 1.3$ and $\bar{l}_{4}=4.3 \pm 0.9$. $\bar{l}_{6}=$ $16.6 \pm 0.4$ is fit to the experimental value of $\left\langle r^{2}\right\rangle$. Note that with the phase shift data one can only determine the difference $\bar{l}_{2}-\bar{l}_{1}$ which is about 6 . Using Eq. (2.4), the curvature is then

$$
c_{V}^{\pi}=4.00 \pm 0.50 \mathrm{GeV}^{-4}, \quad\left\langle r^{4}\right\rangle=0.73 \pm 0.09 \mathrm{fm}^{4}
$$

in good agreement with our cross-check Breit-Wigner values and other studies. The quantity depending solely on the phase shift is then $\tilde{c}_{V}^{\pi}=2.13 \pm 0.42 \mathrm{GeV}^{-4}$. These values are to be considered as our results at the physical pion mass.

\section{Varying the pion mass}

In fig. 2 we show the dependence of the quartic radius and $\tilde{c}_{V}$ as function of the pion mass, as reported in [3]. We also plot the very recent lattice computation of [5] that shows excellent agreement with our results, that can also be used for future lattice extrapolations in the quark mass. Note that the lattice simulations came out after our prediction. The excellent agreement is nontrivial, and should be regarded as a strong support of our analysis.

Two more comments are of interest for lattice practitioners. The first is the observation that, taking derivatives of eq.(2.3), one can easily obtain further relations analogous to eq.(2.4). This family of equations allows, having at hand a computation of the spacelike form factor, and having extracted the coefficients of its Taylor expansion at low momentum (averages of higher powers of radii), to access higher integral (inverse) moments of the phase shift in pion-pion scattering, which is technically more involved to extract. 

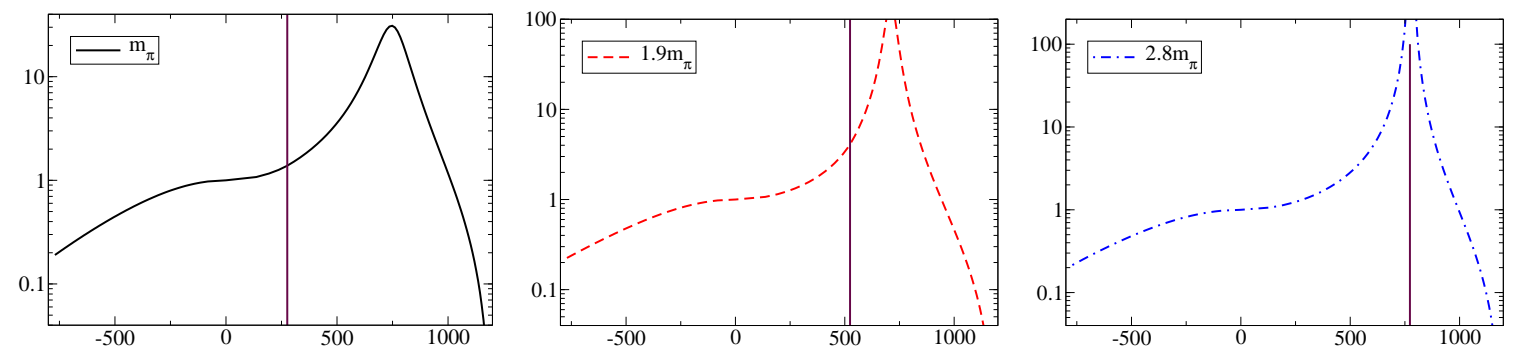

Figure 3: $m_{\pi}$ evolution of the pion form factor in the Omnès representation with the IAM. Left: physical pion mass $m_{\pi}=m_{\pi}^{\text {phys }}$. Middle: just under twice $m_{\pi}^{\text {phys }}$. Right: just under three times $m_{\pi}^{\text {phys }}$. The $\rho$ becomes narrower and taller as the decay threshold $2 m_{\pi}$ (vertical bar) approaches it.

Our second point concerns the reach of quark-mass extrapolation in the lattice. In fig. 3 we have plotted the evolution of the spacelike and timelike form factors as function of the pion mass. Since the $\rho$ mass, controlled by $\Lambda_{Q C D}$ grows only linearly with the quark mass, the threshold for $\rho \rightarrow \pi \pi$ (the vertical bar in the figure) increases faster than the resonance mass, eventually closing the phase space for the decay. But the factor of $\sqrt{\left(s-4 m_{\pi}^{2}\right)^{3}}$, that causes a non-analyticity in $s$, also produces one as a function of $m_{\pi}$. This propagates, through the Omnès representation, to the form factor. Hence we conclude that the dependence of any moment of the form factor on the pion mass is an analytic function around the physical mass, but for high enough $m_{\pi}$, a kink is found that spoils the usual polynomial or chiral extrapolation of lattice data.

This phenomenon is generic and occurs whenever a resonance dives under its decay threshold, the intensity of the kink depending on the width of the resonance at the physical point (for couplings that are weakly dependent on the pion mass). We are preparing a further work with a complete investigation [8].

We thank the organizers of EFT09 for the opportunity to discuss this work, supported by grants FPA2007-29115-E, MCYT FPA 2008-00592/FPA, FIS2008-01323 (Spain), and by the Helmholtz Association through funds provided to the virtual institute "Spin and strong QCD" (VH-VI-231).

\section{References}

[1] J. Gasser and U. G. Meissner, Nucl. Phys. B 357 (1991) 90.

[2] C. Hanhart, J. R. Pelaez and G. Rios, Phys. Rev. Lett. 100 (2008) 152001; P. C. Bruns and U. G. Meissner, Eur. Phys. J. C 40 (2005) 97; D. Djukanovic, J. Gegelia, A. Keller and S. Scherer, arXiv:0902.4347 [hep-ph];

[3] Feng-Kun Guo et al, arXiv:0812.3270 [hep-ph], submitted to Physics Letters B.

[4] G. Abbas, B. Ananthanarayan and S. Ramanan, arXiv:0903.4297 [hep-ph].

[5] R. Frezzotti, V. Lubicz and S. Simula, arXiv:0812.4042 [hep-lat].

[6] J. Bijnens, G. Colangelo and P. Talavera, JHEP 9805 (1998) 014 [arXiv:hep-ph/9805389].

[7] A. Dobado, M. J. Herrero and T. N. Truong, Phys. Lett. B 235 (1990) 134.

[8] F.K.Guo et al., work in preparation. 